\title{
Interval estimation for continuous-time switched linear systems ${ }^{\star}$
}

\author{
Haifa Ethabet $^{\mathrm{a}}$, Djahid Rabehi ${ }^{\mathrm{b}}$, Denis Efimov ${ }^{\mathrm{c}}$, Tarek Raïssi ${ }^{\mathrm{d}}$ \\ ${ }^{a}$ Research Laboratory Modeling, Analysis and Control of Systems (MACS) LR16ES22, National Engineering School of Gabes \\ (ENIG), University of Gabes 6029 Gabes, Tunisia. \\ ${ }^{\mathrm{b}}$ Univ. Orléans, INSA Center Val de Loire (CVL), PRISME, EA 4229, Orléans, France. \\ ${ }^{\mathrm{c}}$ Inria, Non-A team, Parc Scientifique de la Haute Borne, 40 av. Halley, 59650 Villeneuve d'Ascq, France. \\ ${ }^{\mathrm{d}}$ Conservatoire National des Arts et Metiers (CNAM), Cedric - lab 292, Rue St-Martin, 75141 Paris Cedex 03, France.
}

\begin{abstract}
This paper deals with the design of interval observers for switched linear systems (SLS), a class of hybrid systems. Under the assumption that the disturbances and the measurement noise are bounded, upper and lower bounds for the state are calculated. New conditions of cooperativity in discrete-time instants are firstly proposed. Then, some techniques for interval estimation are developed in continuous-time. It is shown that it is possible to calculate the observer gains making the estimation error dynamics cooperative and stable via some change of coordinates under arbitrary switching sequences. The performances of the developed techniques are illustrated through numerical examples.
\end{abstract}

Key words: Interval observer; Cooperative dynamics; Continuous-time switched systems; Stability.

\section{Introduction}

Switched systems are very flexible modeling tools, which appear in several fields such as networked control systems, electrical devices and congestion modeling [18]. These systems are one of the most important classes of Hybrid Dynamical Systems (HDS). They consist of a set of continuous dynamical systems and a switching rule orchestrates among them, their detailed description can be found in various monographs like [5], [18].

In order to study the stability of SLS, specific results have been developed. For example, in [15] a common Lyapunov function yields sufficient conditions for the global asymptotic stability. However, it may not always be possible to get this common function. Therefore,

\footnotetext{
* This work was partially supported by the Government of Russian Federation (Grant 074-U01) and the Ministry of Education and Science of Russian Federation (Project 14.Z50.31.0031).

Email addresses: thabethaifa@gmail.com (Haifa Ethabet), djahid.rabehi@gmail.com (Djahid Rabehi), Denis.Efimov@inria.fr (Denis Efimov), tarek.raissi@cnam.fr (Tarek Raïssi).
}

multiple Lyapunov functions were proposed for instance in [18]. Approaches based on restrictions on admissible rates of commutation, like average dwell time, have also been studied to ensure the stability of SLS(see [14], [19] and the references therein).

As far as the stability problem is widely concerned, it is worth pointing out that the state is not always directly measured but may be estimated from the input and the output of the process. State estimation of those systems has received considerable attention over past decades. Meanwhile, some recent researches have been carried out in this domain. In the case of SLS with state jumps, necessary and sufficient conditions for the observability have been established based on geometric approach [31]. In [29], [1] sliding mode observers have been designed to estimate the continuous and discrete states for the switched system in observability canonical form. In [34] the authors have considered the problem of existence of Luenberger-type observers [20] in the case when the activated observer is time delayed compared to the activated subsystem of the switched system.

The estimation problem becomes much more involved if we consider systems subject to model and/or signals 
uncertainties. Therefore, state estimation approaches based on the set-membership [23] and interval observers get more attention for uncertain systems (a survey is given in [7]). According to the interval observers theory, the uncertainties are assumed to be unknown but bounded with a priori known bounds. In the literature, interval observers are applied to several classes of nonlinear systems [27], [23], discrete-time systems [22], [6] and linear systems [10], [17].

For HDS, an interval observer has been applied to the estimation of linear impulsive systems [3], while the extension of those estimators to switched systems has not been fully considered in the literature. To the best of the authors knowledge, only a preliminary work has been developed in [16], [13], [12] with the strong assumption that there exist observer gains providing the cooperativity and stability conditions simultaneously. Unfortunately, this assumption is rather restrictive and rarely verified in practice and different attempts to overcome this restriction have been performed in [26], [8], and the present paper is a journal extension of the latter works.

In this work, the main contribution is to design interval estimators for SLS subject to disturbances. The measurement noise and the state disturbance are assumed to be unknown but bounded with known bounds. Discretetime and continuous-time interval estimations have been considered. In the first approach, the cooperativity property of the observation error is ensured, under some approximations, through judicious observer gains computation, and the stability analysis is verified by the feasibility of matrix inequalities with respect to a matrix variable given in a quadratic Lyapunov function. While in the second one, the stability and cooperativity conditions are given in terms of Linear Matrix Inequalities (LMIs). It will be shown that the constructive methodologies can be applied for a large class of SLS.

The paper has the following structure. Some preliminaries are described in Section 2. The main results of designing the interval observers are developed in Section 3. Simulation results are shown in Section 4 to illustrate the efficiency of the proposed methods. Section 5 concludes the paper.

\section{Preliminaries}

\subsection{Notations}

The sets of real and natural numbers are denoted by $\mathbb{R}$ and $\mathbb{N}$ respectively. Denote the sequence of integers $\{1, \ldots, N\}$ by $\overline{1, N} . E_{p}$ denotes a $(p \times 1)$ vector whose elements are equal to 1 . $I$ is the identity matrix of proper dimension. For a matrix $P=P^{T}$, the relation $P \prec 0$ $(P \succ 0)$ means that the matrix $P \in \mathbb{R}^{n \times n}$ is negative (positive) definite. Denote by $\underline{x}$ and $\bar{x}$ the lower and upper bounds of a variable $x$ such that $\underline{x} \leq x \leq \bar{x}$. $|\cdot|$ denotes the elementwise absolute value of a vector $x \in \mathbb{R}^{n}$ resp. a matrix $A \in \mathbb{R}^{n \times m}$. The relation $\leq$ should be interpreted elementwise for vectors as well as for matrices, i.e. $A=\left(a_{i, j}\right) \in \mathbb{R}^{p \times m}$ and $B=\left(b_{i, j}\right) \in \mathbb{R}^{p \times m}$ such that $A \geq B$ if and only if, $a_{i, j} \geq b_{i, j}$ for all $i \in \overline{1, p}, j \in \overline{1, m}$. $\operatorname{Diag}(\lambda)$ denotes a diagonal matrix with the elements of the vector $\lambda$ on the main diagonal.

For a matrix $A \in \mathbb{R}^{m \times n}$, define $A^{+}=\max \{0, A\}$ and $A^{-}=A^{+}-A$.

Lemma 1 [11] Let $\delta>0$ be a scalar and $S \in \mathbb{R}^{n \times n}$ be a symmetric positive definite matrix, then

$$
2 x^{T} y \leq \frac{1}{\delta} x^{T} S x+\delta y^{T} S^{-1} y \quad x, y \in \mathbb{R}^{n} .
$$

\subsection{Cooperativity}

Definition 1 [24] A matrix $A=\left(a_{i, j}\right) \in \mathbb{R}^{n \times n}$ is said to be Metzler if all its off-diagonal elements are nonnegative i.e. $a_{i, j} \geq 0, \forall(i, j), i \neq j$. It is said to be nonnegative if all the entries are nonnegative: $A \geq 0$.

Lemma 2 [10], [9] Consider the system described by:

$$
\dot{x}(t)=A x(t)+u(t), x(0)=x_{0}
$$

The system (2) is said to be cooperative if $A$ is a Metzler matrix and $u(t) \geq 0$. For any initial condition $x_{0} \geq 0$ the solution of (2) satisfies $x(t) \geq 0, \forall t \geq 0$.

\subsection{Interval relations}

Property 1 [2] Given a matrix $A \in \mathbb{R}^{n \times n}$. A is Metzler if there exists a diagonal matrix $S \in \mathbb{R}_{+}^{n}$ such that

$$
A+S>0
$$

Lemma 3 [2] Let $x \in \mathbb{R}^{n}$ be a vector satisfying $\underline{x} \leq x \leq$ $\bar{x}$ and $A \in \mathbb{R}^{m \times n}$ be a constant matrix, then

$$
A^{+} \underline{x}-A^{-} \bar{x} \leq A x \leq A^{+} \bar{x}-A^{-} \underline{x} .
$$

\subsection{Basic interval observer theory}

For the sake of clarity, let us recall some definitions of interval observers [7]. Consider the following system:

$$
\left\{\begin{array}{l}
\dot{x}=A x+\phi(t) \\
y=C x
\end{array}\right.
$$

where $\phi$ is a continuous function of time. Assume that there exist two known functions $\bar{\phi}$ and $\phi: \mathbb{R} \rightarrow \mathbb{R}^{n}$ such that $\phi(t) \leq \phi(t) \leq \bar{\phi}(t), \forall t \geq 0$.

Interval observers compute a guaranteed set that contains admissible values for the state vector of the system 
consistent with the output measurements. Upper and lower bounds of the set are given in the following theorem.

Theorem 1 [10] Let $\underline{x}_{0} \leq x_{0} \leq \bar{x}_{0}$. If there exists a gain $K$ such that $(A-K C)$ is Metzler and Hurwitz then

$$
\left\{\begin{array}{l}
\dot{\bar{x}}=A \bar{x}+\bar{\phi}+K(y-C \bar{x}) \\
\underline{\dot{x}}=A \underline{x}+\underline{\phi}+K(y-C \underline{x})
\end{array}\right.
$$

is an interval observer with $\underline{x}(t) \leq x(t) \leq \bar{x}(t), \forall t \geq 0$.

\subsection{Stability}

Consider the following continuous-time SLS:

$$
\dot{x}(t)=A_{\sigma(t)} x(t), \sigma(t) \in \mathcal{I}=\overline{1, N}, N \in \mathbb{N}
$$

where $x \in \mathbb{R}^{n}$ is the state, $N$ is the number of linear subsystems, the finite set $\mathcal{I}$ is an index set and it stands for the collection of subsystems $A_{q} \in \mathbb{R}^{n \times n}, \forall q \in \mathcal{I}$. The switching between the subsystems is ensured via a switching signal, a piecewise constant function, $\sigma(t)$ : $\mathbb{R}_{+} \rightarrow \mathcal{I}$. The index $q=\sigma(t)$ specifies, at each instant of time, the system that currently being followed.

Lemma 4 [18] Let $S \in \mathbb{R}^{n \times n}$ be a symmetric positive definite matrix that satisfies the LMIs

$$
A_{q}^{T} S+S A_{q} \prec 0, q \in \mathcal{I}=\overline{1, N}
$$

Then $V(x)=x^{T} S x$ is a Common Quadratic Lyapunov Function (CQLF) for the system (7).

This lemma establishes conditions of the internal stability (without taking into account the effect of external inputs). For SLS with inputs and properly assigned dwell-time switching signals, the overall system is inputto-state stable (ISS) if the individual subsystems are ISS [33].

\section{Main results}

In this section several interval observers are designed for SLS that would satisfy both the stability and the cooperativity conditions. Consider a continuous-time switched system

$$
\left\{\begin{array}{l}
\dot{x}(t)=A_{\sigma(t)} x(t)+B_{\sigma(t)} u(t)+w(t) \\
y_{m}(t)=C_{\sigma(t)} x(t)+v(t)
\end{array}, \sigma(t) \in \mathcal{I}\right.
$$

where $x \in \mathbb{R}^{n}, u \in \mathbb{R}^{m}, y_{m} \in \mathbb{R}^{p}, w \in \mathbb{R}^{n}, v \in \mathbb{R}^{p}$ are the state vector, the input, the output, the disturbance and the measurement noise respectively. $q=\sigma(t)$ is the index of the active subsystem and assumed to be known. $A_{q}, B_{q}$ and $C_{q}$ are constant matrices of appropriate dimensions.

The aim is to derive two signals $\underline{x}(t)$ and $\bar{x}(t)$ such that $\underline{x}(t) \leq x(t) \leq \bar{x}(t), \forall t \geq 0$ holds despite the disturbances and uncertainties provided that $\underline{x}_{0} \leq x_{0} \leq \bar{x}_{0}$ is satisfied. This section has three parts. First, a basic interval observer will be introduced under the most restrictive assumptions. Second and third parts will present two different ways of these hypothesises relaxation: either passing to discrete-time estimates using interesting properties of matrix exponential observed by Trotter, or via a change of coordinates avoiding state jumps.

\subsection{A basic interval observer}

Let us first consider the simple structure of an interval observer for (9). It is based on the classical Luenberger observer [20] with proper assumptions that the estimation error dynamics is cooperative [27], [21], [28], and [7].

Assumption 1 The measurement noise and the state disturbance are assumed to be unknown but bounded with a priori known bounds such that

$$
-\bar{w}(t) \leq w(t) \leq \bar{w}(t),|v(t)| \leq \bar{V} E_{p}, \quad \forall t \geq 0
$$

where $\bar{w} \in \mathbb{R}^{n}$ and $\bar{V}$ is a positive scalar.

Assumption 2 There exist gains $L_{q}$ such that the matrices $\left(A_{q}-L_{q} C_{q}\right)$ are Metzler for all $q \in \mathcal{I}$.

The matrices $L_{q}(q \in \mathcal{I})$ denote the observer gains associated with each subsystem $q$.

A candidate interval observer structure for computing $\bar{x}$ and $\underline{x}$ is described, as in Theorem 1, by:

$$
\left\{\begin{array}{l}
\dot{\bar{x}}=\left(A_{q}-L_{q} C_{q}\right) \bar{x}+B_{q} u+\bar{w}+L_{q} y_{m}+\left|L_{q}\right| \bar{V} E_{p} \\
\dot{\dot{x}}=\left(A_{q}-L_{q} C_{q}\right) \underline{x}+B_{q} u-\bar{w}+L_{q} y_{m}-\left|L_{q}\right| \bar{V} E_{p}
\end{array}\right.
$$

The following theorem gives the conditions for achieving the desired design goal.

Theorem 2 Given $\underline{x}_{0}, \bar{x}_{0} \in \mathbb{R}^{n}$. Let Assumption 1 be satisfied. If there exist $\lambda, \eta \in \mathbb{R}^{n}, \lambda>0, \eta>0$, matrices $W_{q} \in \mathbb{R}^{n \times n}$ and positive scalars $\alpha_{q}, \forall q \in \mathcal{I}$ such that

$$
\left\{\begin{array}{l}
A_{q}^{T} S+S A_{q}-C_{q}^{T} W_{q}^{T}-W_{q} C_{q}+\alpha_{q} S \prec 0 \\
A_{q}^{T} S-C_{q}^{T} W_{q}^{T}+\operatorname{diag}(\eta) \geq 0 \\
S=\operatorname{diag}(\lambda)
\end{array}\right.
$$

then the system (11) satisfies with the system (9) the inclusion

$$
\underline{x}(t) \leq x(t) \leq \bar{x}(t)
$$

provided that $\underline{x}_{0} \leq x_{0} \leq \bar{x}_{0}$, where $L_{q}=S^{-1} W_{q}$. 
PROOF. Let $\bar{e}(t)=\bar{x}-x$ and $\underline{e}(t)=x-\underline{x}$ be respectively the upper and the lower observation errors. From (9) and (11) the dynamics of the interval estimation errors are given by:

$\dot{\bar{e}}(t)=\dot{\bar{x}}-\dot{x}=\left(A_{q}-L_{q} C_{q}\right) \bar{e}(t)+\bar{\Gamma}_{q}$

$\underline{\dot{e}}(t)=\dot{x}-\underline{\dot{x}}=\left(A_{q}-L_{q} C_{q}\right) \underline{e}(t)+\underline{\Gamma}_{q}$

where $\bar{\Gamma}_{q}=\bar{w}-w+L_{q} v+\left|L_{q}\right| \bar{V} E_{p}$

$$
\underline{\Gamma}_{q}=w+\bar{w}-L_{q} v+\left|L_{q}\right| \bar{V} E_{p}
$$

If (12) is satisfied, then multiplying the second LMI in (12) by $\operatorname{diag}(\lambda)^{-1}$ yields $A_{q}^{T} \operatorname{Sdiag}(\lambda)^{-1}-$ $C_{q}^{T} W_{q}^{T} \operatorname{diag}(\lambda)^{-1}+\operatorname{diag}(\eta) \operatorname{diag}(\lambda)^{-1} \geq 0$. Since $S=\operatorname{diag}(\lambda)$ and $L_{q}=S^{-1} W_{q}$, it follows that $A_{q}^{T}-C_{q}^{T} L_{q}^{T}+\operatorname{diag}(\eta) \operatorname{diag}(\lambda)^{-1} \geq 0$ or equivalently that $\left(A_{q}-L_{q} C_{q}\right)$ are Metzler $\forall q \in \mathcal{I}$. Due to $A s$ sumption 1 , it is clear that $\bar{\Gamma}_{q}$ and $\underline{\Gamma}_{q}$ are nonnegative $\forall q \in \mathcal{I}$ and $t \geq 0$. Based on Lemma 2, it follows that $\underline{x}(t) \leq x(t) \leq \bar{x}(t)$ is satisfied, provided that $\underline{x}_{0} \leq \bar{x}_{0} \leq \bar{x}_{0}$.

To prove that $\underline{x}(t), \bar{x}(t)$ are bounded, consider the Lyapunov function $V(\bar{e})=\bar{e}(t)^{T} S \bar{e}(t)$ where $S=S^{T} \succ 0$. The derivative of $V$ is given by:

$$
\begin{aligned}
\dot{V}(\bar{e})= & \dot{\bar{e}}^{T} S \bar{e}+\bar{e}^{T} S \dot{\bar{e}} \\
= & \bar{e}^{T}\left[\left(A_{q}-L_{q} C_{q}\right)^{T} S+S\left(A_{q}-L_{q} C_{q}\right)\right] \bar{e}-2 \bar{e}^{T} S w \\
& +2 \bar{e}^{T} S L_{q} v+2 \bar{e}^{T} S \bar{w}+2 \bar{e}^{T} S\left|L_{q}\right| \bar{V} E_{p}
\end{aligned}
$$

From Lemma 1, it is clear that

$$
\begin{gathered}
2 \bar{e}^{T} S w \leq \frac{1}{\delta_{q}} \bar{e}^{T} S \bar{e}+\delta_{q} w^{T} S w \\
2 \bar{e}^{T} S L_{q} v \leq \frac{1}{\delta_{q}} \bar{e}^{T} S \bar{e}+\delta_{q} v^{T} L_{q}^{T} S L_{q} v \\
2 \bar{e}^{T} S \bar{w} \leq \frac{1}{\delta_{q}} \bar{e}^{T} S \bar{e}+\delta_{q} \bar{w}^{T} S \bar{w} \\
2 \bar{e}^{T} S\left|L_{q}\right| \bar{V} E_{p} \leq \frac{1}{\delta_{q}} \bar{e}^{T} S \bar{e}+\delta_{q} E_{p}^{T} \bar{V}\left|L_{q}\right|^{T} S\left|L_{q}\right| \bar{V} E_{p}
\end{gathered}
$$

Let $W_{q}=S L_{q}$, the combination of (15)-(19) leads to $\dot{V}(\bar{e}) \leq \bar{e}^{T} \mathcal{B}_{1} \bar{e}+\mathcal{C}_{1}$ where

$$
\begin{aligned}
\mathcal{B}_{1}= & A_{q}^{T} S+S A_{q}-C_{q}^{T} W_{q}^{T}-W_{q} C_{q}+\frac{2}{\delta_{q}} S \\
\mathcal{C}_{1}= & w^{T}\left[-\delta_{q} S\right] w+\bar{w}^{T}\left[\delta_{q} S\right] \bar{w}+v^{T}\left[\delta_{q} L_{q}^{T} S L_{q}\right] v \\
& +E_{p}^{T}\left[\delta_{q} \bar{V}\left|L_{q}\right|^{T} S\left|L_{q}\right| \bar{V}\right] E_{p}
\end{aligned}
$$

Similarly, the derivative of the common Lyapunov function for the lower estimation error yields:

$$
\begin{aligned}
\dot{V}(\underline{e}) & =\underline{e}^{T}\left[\left(A_{q}-L_{q} C_{q}\right)^{T} S+S\left(A_{q}-L_{q} C_{q}\right)\right] \underline{e} \\
& +2 \underline{e}^{T} S w+2 \underline{e}^{T} S \bar{w}-2 \underline{e}^{T} S L_{q} v+2 \underline{e}^{T} S\left|L_{q}\right| \bar{V} E_{p} \\
& \leq \underline{e}^{T} \mathcal{B}_{1} \underline{e}+\mathcal{C}_{2}
\end{aligned}
$$

where $\mathcal{C}_{2}=w^{T}\left[\delta_{q} S\right] w+\bar{w}^{T}\left[\delta_{q} S\right] \bar{w}-v^{T}\left[\delta_{q} L_{q}^{T} S L_{q}\right] v$

$$
+E_{p}^{T}\left[\delta_{q} \bar{V}\left|L_{q}\right|^{T} S\left|L_{q}\right| \bar{V}\right] E_{p}
$$

From the first equation of (12), it is assumed that $\mathcal{B}_{1} \prec$ 0 . Taking into account that $\left(A_{q}-L_{q} C_{q}\right)$ are Metzler, then according to [2] the matrix $S$ is diagonal and can be written as $S=\operatorname{diag}(\lambda), \lambda>0$. In addition, since noises and disturbances are bounded it follows that $\mathcal{C}_{1}$ is bounded. Therefore the system (13) is ISS stable and the error $\bar{e}$ is bounded. The same arguments show that the error $\underline{e}$ is also bounded.

Remark 1 The system (11) is initialized with the initial conditions $\underline{x}_{0}$ and $\bar{x}_{0}$ for the first active subsystem. At the switching time instant $t_{i+1}$, the output of the previous active subsystem $\left(q=\sigma\left(t_{i}\right)\right)$ is used to initialize (11) with the subsystem $\left(q=\sigma\left(t_{i+1}\right)\right)$.

The LMI (12) is formulated with the aim to find the observer gains $L_{q}$ ensuring the system stability, it can be reformulated in order to provide a minimization of the interval estimation error $\bar{x}-\underline{x}$ as in [2].

\subsection{Trotter approximation based interval observer}

In this section an interest is carried to discrete time observers. For brevity of presentation, we will assume that $\mathcal{I}=\{1,2\}$. The generic case with $N \geq 2$ can be treated similarly [30]. The conditions of cooperativity in Assumption 2 will be relaxed by computing gain matrices $L_{q} \in \mathbb{R}^{n \times p}, q \in \mathcal{I}$ that satisfy $\sum_{i=1}^{2}\left(A_{i}+L_{i} C_{i}\right)$ is Metzler. Under this condition, which would be more straightforward to be fulfilled, the following Trotter formula result can be used.

Theorem 3 [32] For two given matrices $B, C \in \mathbb{R}^{n \times n}$, the exponentials of $B$ and $C$ are related to that of $B+C$ as follows

$$
\lim _{m \rightarrow \infty}\left(e^{\frac{B}{m}} e^{\frac{C}{m}}\right)^{m}=e^{(B+C)} .
$$

The Trotter result can be used to evaluate $e^{A}$ by splitting $A$ into $B+C$ and then using the approximation $e^{A} \simeq$ $\left(e^{\frac{B}{m}} e^{\frac{C}{m}}\right)^{m}$ for a sufficiently big $m \geq 0$. From the system (9) we have

$$
\begin{aligned}
\dot{x}(t)= & A_{q} x(t)+B_{q} u(t)+w(t) \\
= & \left(A_{q}-L_{q} C_{q}\right) x(t)+w(t)+B_{q} u(t) \\
& +L_{q} y_{m}(t)-L_{q} v(t), \quad q \in \mathcal{I}
\end{aligned}
$$

In this subsection it will be considered that the switching is periodical and $t_{k+1}-t_{k}=\tau$ for some period $\tau>0$. The system's state between two switching times can be calculated analytically from the equation (22) 
$x\left(t_{k+1}\right)=e^{\tilde{A}_{\sigma\left(t_{k}\right)}\left(t_{k+1}-t_{k}\right)} x\left(t_{k}\right)+\int_{t_{k}}^{t_{k+1}} e^{\tilde{A}_{\sigma\left(t_{k}\right)}\left(t_{k+1}-s\right)}$

$\left[B_{\sigma\left(t_{k}\right)} u(s)+L_{\sigma\left(t_{k}\right)} y_{m}(s)+\omega(s)-L_{\sigma\left(t_{k}\right)} v(s)\right] d s$

where $\tilde{A}_{\sigma\left(t_{k}\right)}=A_{\sigma\left(t_{k}\right)}-L_{\sigma\left(t_{k}\right)} C_{\sigma\left(t_{k}\right)}$ and $\sigma\left(t_{k}\right)$ corresponds to the activated system. After switching $2 m$ times between subsystems (22), the state variables will be obtained through the analytical solution

$$
\begin{aligned}
x(T) & =\prod_{k=0}^{2 m-1} e^{\tilde{A}_{\sigma\left(t_{k}\right)} \tau} x\left(t_{0}\right) \\
& +\Lambda\left(y_{m}(T), u(T)\right)+\Pi(w(T), v(T))
\end{aligned}
$$

where $T=2 m \tau$,

$$
\begin{aligned}
& \Lambda\left(y_{m}(T), u(T)\right)=\sum_{k=1}^{2 m}\left[\prod_{j=k}^{2 m-1}\left(e^{\tilde{A}_{\sigma\left(t_{j}\right)} \tau}\right) \times\right. \\
& \left.\int_{t_{k-1}}^{t_{k}} e^{\tilde{A}_{\sigma\left(t_{k-1}\right)}\left(t_{k}-s\right)}\left(B_{\sigma\left(t_{k-1}\right)} u(s)+L_{\sigma\left(t_{k-1}\right)} y_{m}(s)\right) d s\right]
\end{aligned}
$$

and

$$
\begin{gathered}
\Pi(w(T), v(T))=\sum_{k=1}^{2 m}\left[\prod_{j=k}^{2 m-1}\left(e^{\tilde{A}_{\sigma\left(t_{j}\right)} \tau}\right)\right. \\
\left.\times \int_{t_{k-1}}^{t_{k}} e^{\tilde{A}_{\sigma\left(t_{k-1}\right)}\left(t_{k}-s\right)}\left(w(s)-L_{\sigma\left(t_{k-1}\right)} v(s)\right) d s\right]
\end{gathered}
$$

Using the fact that $\tilde{A}_{\sigma\left(t_{k}\right)} \in\left\{\tilde{A}_{1}, \tilde{A}_{2}\right\}$,

$$
\prod_{k=0}^{2 m-1} e^{\tilde{A}_{\sigma\left(t_{k}\right)} \tau}=\left(e^{\tilde{A}_{1} \tau} e^{\tilde{A}_{2} \tau}\right)^{m}=\left(e^{\tilde{A}_{1} \frac{T}{2 m}} e^{\tilde{A}_{2} \frac{T}{2 m}}\right)^{m}
$$

Using the Trotter formula (21) we know

$$
\lim _{m \rightarrow \infty}\left(e^{\tilde{A}_{1} \frac{T}{2 m}} e^{\tilde{A}_{2} \frac{T}{2 m}}\right)^{m}=e^{\left(\tilde{A}_{1}+\tilde{A}_{2}\right) \frac{T}{2}}
$$

Coupling the equation (23) and the formula (27) leads

$$
\begin{aligned}
x((l+1) T) & =\left(e^{\left(\tilde{A}_{1}+\tilde{A}_{2}\right) \frac{T}{2}}-\Delta\right) x(l T) \\
& +\Lambda\left(y_{m}((l+1) T), u((l+1) T)\right) \\
& +\Pi(w((l+1) T), v((l+1) T))
\end{aligned}
$$

where $\Delta=\left[e^{\left(\tilde{A}_{1}+\tilde{A}_{2}\right) \frac{T}{2}}-\left(e^{\tilde{A}_{1} \frac{T}{2 m}} e^{\tilde{A}_{2} \frac{T}{2 m}}\right)^{m}\right]$.

Due to Trotter result we know that the matrix $\Delta$ converges to zero by increasing $m$. Then the interval observer for (28) can be designed at discrete time instants in the following form:

$$
\begin{aligned}
\underline{x}((l+1) T)= & e^{\left(\tilde{A}_{1}+\tilde{A}_{2}\right) \frac{T}{2}} \underline{x}(l T)+\Delta^{-} \underline{x}(l T)-\Delta^{+} \bar{x}(l T) \\
& +\underline{\Pi}_{2 m}+\Lambda\left(y_{m}((l+1) T), u((l+1) T)\right), \\
\bar{x}((l+1) T)= & e^{\left(\tilde{A}_{1}+\tilde{A}_{2}\right) \frac{T}{2}} \bar{x}(l T)+\Delta^{-} \bar{x}(l T)-\Delta^{+} \underline{x}(l T) \\
& +\bar{\Pi}_{2 m}+\Lambda\left(y_{m}((l+1) T), u((l+1) T)\right)
\end{aligned}
$$

where $\underline{\Pi}_{2 m}, \bar{\Pi}_{2 m}$ are calculated by

$$
\begin{aligned}
& \underline{\Pi}_{k}= \begin{cases}\Psi_{k}^{+} \underline{\Pi}_{k-1}-\Psi_{k}^{-} \bar{\Pi}_{k-1}+\underline{D}_{k} & \text { for } k \geq 2 \\
\underline{D}_{k} & \text { for } k=1\end{cases} \\
& \bar{\Pi}_{k}= \begin{cases}\Psi_{k}^{+} \bar{\Pi}_{k-1}-\Psi_{k}^{-} \underline{\Pi}_{k-1}+\bar{D}_{k} & \text { for } k \geq 2 \\
\bar{D}_{k} & \text { for } k=1\end{cases}
\end{aligned}
$$

where $\Psi_{k}=\left(e^{\left.\tilde{A}_{\sigma\left(t_{k-1}\right.}\right)^{\tau}}\right)$ and the quantities $\underline{D}_{k}, \bar{D}_{k}$ will be defined by expressions (31) in Proposition 1 below.

Assumption 3 There exist two matrices $L_{1}, L_{2} \in \mathbb{R}^{n \times p}$ such that the matrix $A_{1}-L_{1} C_{1}+A_{2}-L_{2} C_{2}$ is Metzler.

It has been shown in [24], [25] that the matrix $e^{A t} \geq 0$ is nonnegative if and only if $A \in \mathbb{R}^{n \times n}$ is a Metzler matrix. Thereby, Assumption 3 allows us to conclude that the matrix $e^{\left(\tilde{A}_{1}+\tilde{A}_{2}\right) \frac{T}{2}}$ is nonnegative for all $T \geq 0$.

Assumption 4 There exist two matrices $L_{1}, L_{2} \in$ $\mathbb{R}^{n \times p}$, a symmetric positive definite matrix $S_{\Gamma} \in \mathbb{R}^{2 n \times 2 n}$ and a scalar $m \in \mathbb{N}$ such that the nonnegative matrix

$$
\Gamma=\left(\begin{array}{cc}
e^{\left(\tilde{A}_{1}+\tilde{A}_{2}\right) \frac{T}{2}}+\Delta^{-} & \Delta^{+} \\
\Delta^{+} & e^{\left(\tilde{A}_{1}+\tilde{A}_{2}\right) \frac{T}{2}}+\Delta^{-}
\end{array}\right)
$$

satisfies the matrix inequality

$$
\Gamma^{T} S_{\Gamma} \Gamma-S_{\Gamma} \prec 0
$$

with respect to the matrix variable $S_{\Gamma}$.

Proposition 1 Under Assumption 1 the inequality $\underline{D}_{k} \leq D_{k} \leq \bar{D}_{k}$ is satisfied, where

$$
\begin{aligned}
\underline{D}_{k} & =\int_{t_{k-1}}^{t_{k}}\left(\left(e^{\tilde{A}_{\sigma\left(t_{k-1}\right)}\left(t_{k}-s\right)}\right)^{+} \underline{w}(s)-\left(e^{\tilde{A}_{\sigma\left(t_{k-1}\right)}\left(t_{k}-s\right)}\right)^{-}\right. \\
\left.\bar{w}(s)-\left|e^{\tilde{A}_{\sigma\left(t_{k-1}\right)}\left(t_{k}-s\right)} L_{\sigma\left(t_{k-1}\right)}\right| E_{p} \bar{V}\right) d s & \\
D_{k}= & \int_{t_{k-1}}^{t_{k}} e^{\tilde{A}_{\sigma\left(t_{k-1}\right)}\left(t_{k}-s\right)}\left(w(t)-L_{\sigma\left(t_{k-1}\right)} v(t)\right) d s, \\
\bar{D}_{k}= & \int_{t_{k-1}}^{t_{k}}\left(\left(e^{\tilde{A}_{\sigma\left(t_{k-1}\right)}\left(t_{k}-s\right)}\right)^{+} \bar{w}(s)-\left(e^{\tilde{A}_{\sigma\left(t_{k-1}\right)}\left(t_{k}-s\right)}\right)^{-}\right. \\
& \left.\underline{w}(s)+\left|e^{\tilde{A}_{\sigma\left(t_{k-1}\right)}\left(t_{k}-s\right)} L_{\sigma\left(t_{k-1}\right)}\right| E_{p} \bar{V}\right) d s .
\end{aligned}
$$


PROOF. Using Lemma 3 (by taking $A=e^{\tilde{A}_{\sigma\left(t_{k-1}\right)}\left(t_{k}-s\right)}$ ) and Assumption 1, and by integrating different terms of the obtained inequality the result is substantiated.

Theorem 4 Let Assumptions 1, 3 and 4 be satisfied and $x \in \mathcal{L}_{\infty}^{n}$, then in the system (28) with the interval observer (29) the relations

$$
\underline{x}(l T) \leq x(l T) \leq \bar{x}(l T), \forall l \in \mathbb{Z}_{+}
$$

are satisfied provided that $\underline{x}(0) \leq x(0) \leq \bar{x}(0)$, in addition the estimates $\underline{x}(l T)$ and $\bar{x}(l \bar{T})$ are bounded.

PROOF. To simplify the calculation, we suppose that each subsystem has been activated one time, hence $m=$ 1 and $T=2 \tau=t_{2}$. So the solution of the system is given by

$$
\begin{aligned}
x\left(t_{2}\right)= & e^{\tilde{A}_{\sigma\left(t_{1}\right)} \tau} e^{\tilde{A}_{\sigma\left(t_{0}\right)} \tau} x\left(t_{0}\right)+e^{\tilde{A}_{\sigma\left(t_{1}\right)} \tau} \int_{t_{0}}^{t_{1}} e^{\tilde{A}_{\sigma\left(t_{0}\right)}\left(t_{1}-s\right)} \times \\
& \left(L_{\sigma\left(t_{0}\right)} y_{m}(s)+B_{\sigma\left(t_{0}\right)} u(s)+w(s)-L_{\sigma\left(t_{0}\right)} v(s)\right) d s \\
& +\int_{t_{1}}^{t_{2}} e^{\tilde{A}_{\sigma\left(t_{1}\right)}\left(t_{2}-s\right)} \times \\
& \left(L_{\sigma\left(t_{1}\right)} y_{m}(s)+B_{\sigma\left(t_{1}\right)} u(s)+w(s)-L_{\sigma\left(t_{1}\right)} v(s)\right) d s \\
= & e^{\tilde{A}_{\sigma\left(t_{1}\right)} \tau} e^{\tilde{A}_{\sigma\left(t_{0}\right)} \tau} x\left(t_{0}\right)+e^{\tilde{A}_{\sigma\left(t_{1}\right)} \tau} D_{1}+D_{2} \\
& +\Lambda\left(y_{m}\left(t_{2}\right), u\left(t_{2}\right)\right) \\
= & e^{\left(\tilde{A}_{1}+\tilde{A}_{2}\right) \frac{T}{2}} x\left(t_{0}\right)+\left(\Delta^{-}-\Delta^{+}\right) x\left(t_{0}\right) \\
& +e^{\tilde{A}_{\sigma\left(t_{1}\right)} \tau} D_{1}+D_{2}+\Lambda\left(y_{m}\left(t_{2}\right), u\left(t_{2}\right)\right)
\end{aligned}
$$

And the estimated bounds are given as

$$
\begin{aligned}
\underline{x}\left(t_{2}\right)= & e^{\left(\tilde{A}_{1}+\tilde{A}_{2}\right) \frac{T}{2}} \underline{x}\left(t_{0}\right)+\Delta^{-} \underline{x}\left(t_{0}\right)-\Delta^{+} \bar{x}\left(t_{0}\right) \\
& +\underline{\Pi}_{2}+\Lambda\left(y_{m}\left(t_{2}\right), u\left(t_{2}\right)\right) \\
\bar{x}\left(t_{2}\right)= & e^{\left(\tilde{A}_{1}+\tilde{A}_{2}\right) \frac{T}{2}} \bar{x}\left(t_{0}\right)+\Delta^{-} \bar{x}\left(t_{0}\right)-\Delta^{+} \underline{x}\left(t_{0}\right) \\
& +\bar{\Pi}_{2}+\Lambda\left(y_{m}\left(t_{2}\right), u\left(t_{2}\right)\right)
\end{aligned}
$$

with $\underline{\Pi}_{2}=\left(e^{\tilde{A}_{\sigma\left(t_{1}\right)} \tau}\right)^{+} \underline{D}_{1}-\left(e^{\tilde{A}_{\sigma\left(t_{1}\right)} \tau}\right)^{-} \bar{D}_{1}+\underline{D}_{2}$,

$$
\bar{\Pi}_{2}=\left(e^{\tilde{A}_{\sigma\left(t_{1}\right)} \tau}\right)^{+} \bar{D}_{1}-\left(e^{\tilde{A}_{\sigma\left(t_{1}\right)} \tau}\right)^{-} \underline{D}_{1}+\bar{D}_{2} .
$$

Using (32), (33) and defining the estimation errors as $\underline{e}\left(t_{2}\right)=x\left(t_{2}\right)-\underline{x}\left(t_{2}\right), \bar{e}\left(t_{2}\right)=\bar{x}\left(t_{2}\right)-x\left(t_{2}\right)$ leads

$\underline{e}\left(t_{2}\right)=e^{\left(\tilde{A}_{1}+\tilde{A}_{2}\right) \frac{T}{2}} \underline{e}\left(t_{0}\right)+\Delta^{+} \bar{e}\left(t_{0}\right)+\Delta^{-} \underline{e}\left(t_{0}\right)+\underline{G}_{1}+\underline{G}_{2}$, $\bar{e}\left(t_{2}\right)=e^{\left(\tilde{A}_{1}+\tilde{A}_{2}\right) \frac{T}{2}} \bar{e}\left(t_{0}\right)+\Delta^{+} \underline{e}\left(t_{0}\right)+\Delta^{-} \bar{e}\left(t_{0}\right)+\bar{G}_{1}+\bar{G}_{2}$.

where

$$
\begin{aligned}
& \underline{G}_{1}=e^{\tilde{A}_{\sigma\left(t_{1}\right) \tau} D_{1}-\left(e^{\tilde{A}_{\sigma\left(t_{1}\right)} \tau}\right)^{+} \underline{D}_{1}-\left(e^{\tilde{A}_{\sigma\left(t_{1}\right)} \tau}\right)^{-} \bar{D}_{1},} \\
& \bar{G}_{1}=\left(e^{\tilde{A}_{\sigma\left(t_{1}\right)} \tau}\right)^{+} \bar{D}_{1}-\left(e^{\tilde{A}_{\sigma\left(t_{1}\right)} \tau}\right)^{-} \underline{D}_{1}-e^{\tilde{A}_{\sigma\left(t_{1}\right)} \tau} D_{1}, \\
& \underline{G}_{2}=D_{2}-\underline{D}_{2}, \\
& \bar{G}_{2}=\bar{D}_{2}-D_{2} .
\end{aligned}
$$

According to Assumption 3 the matrix $e^{\left(\tilde{A}_{1}+\tilde{A}_{2}\right) \frac{T}{2}}$ is nonnegative. By definition $\Delta^{+}, \Delta^{-}$are also nonnegative. By gathering the equations (34) we get

$$
\left[\begin{array}{l}
\underline{e}\left(t_{2}\right) \\
\bar{e}\left(t_{2}\right)
\end{array}\right]=\Gamma\left[\begin{array}{l}
\underline{e}(0) \\
\bar{e}(0)
\end{array}\right]+\left[\begin{array}{l}
\underline{G}_{1}+\underline{G}_{2} \\
\bar{G}_{1}+\bar{G}_{2}
\end{array}\right]
$$

The conditions maintained in Assumption 4 ensure the convergence of the interval observer, that implies boundedness of $\underline{x}(t), \bar{x}(t)$. In addition, since the Assumption 1 holds we can conclude that $\bar{G}_{1} \geq 0, \bar{G}_{2} \geq 0, \underline{G}_{1} \geq$ $0, G_{2} \geq 0$. Therefore the dynamics of the estimation error is cooperative and starting from $\underline{e}(0) \geq 0, \bar{e}(0) \geq 0$ we get that $\underline{e}\left(t_{2}\right) \geq 0, \bar{e}\left(t_{2}\right) \geq 0$. Repeating these steps recurrently the result of theorem can be obtained.

The previous subsections motivate the main results of the next subsection. It worth noting that Assumption 2 can be relaxed. Besides, the second LMI in (12) can be infeasible. Note also that (30) is nonlinear matrix inequality. Some transformation of coordinates will be introduced to alter the system into a cooperative form.

\subsection{Transformation of coordinates}

In this section, the main idea consists in finding a change of coordinates that transforms the observation errors into cooperative forms. The changes of coordinates proposed for instance in [21], [27] for continuous systems can be used to transform the matrices $\left(A_{q}-L_{q} C_{q}\right)$ into a Metzler form. Therefore, let us assume that there exists a non singular transformation matrix $P$ such that, with the new coordinates $z=P x$, the system (9) is transformed into the form

$$
\left\{\begin{array}{l}
\dot{z}=P A_{q} P^{-1} z+P B_{q} u+P w \\
y_{m}=C_{q} P^{-1} z+v
\end{array}\right.
$$

A Luenberger based candidate observer for the system (35) can be written in the new coordinates $z$ as:

$$
\left\{\begin{aligned}
\dot{\bar{z}}= & P\left(A_{q}-L_{q} C_{q}\right) P^{-1} \bar{z}+P B_{q} u+P^{+} \bar{w}-P^{-} \underline{w} \\
& +P L_{q} y_{m}+\left|P L_{q}\right| \bar{V} E_{p} \\
\dot{z}= & P\left(A_{q}-L_{q} C_{q}\right) P^{-1} \underline{z}+P B_{q} u+P^{+} \underline{w}-P^{-} \bar{w} \\
& +P L_{q} y_{m}-\left|P L_{q}\right| \bar{V} E_{p}
\end{aligned}\right.
$$

where $\left\{\begin{array}{l}\bar{z}(0)=P^{+} \bar{x}_{0}-P^{-} \underline{x}_{0} \\ \underline{z}(0)=P^{+} \underline{x}_{0}-P^{-} \bar{x}_{0}\end{array}\right.$

$P$ is the solution of the $N$ Sylvester equations given by

$$
P A_{q}-R_{q} P=Q_{q} C_{q}, \quad Q_{q}=P L_{q}, \forall q \in \mathcal{I}
$$

and $R_{q}=P\left(A_{q}-L_{q} C_{q}\right) P^{-1}$ are Metzler $\forall q \in \mathcal{I}$. Let $\bar{e}_{z}(t)=\bar{z}-z$ be the upper observation error and 
$\underline{e}_{z}(t)=z-\underline{z}$ be the lower one. From systems (35) and (36), the dynamics of the interval estimation errors are given by:

$$
\begin{aligned}
\dot{\bar{e}}_{z}(t)= & \dot{\bar{z}}-\dot{z}=P\left(A_{q}-L_{q} C_{q}\right) P^{-1} \overline{e_{z}} \\
& +\left[\left(P^{+} \bar{w}-P^{-} \underline{w}\right)-P w\right]+\left|P L_{q}\right| \bar{V} E_{p} \\
& +P L_{q} v=R_{q} \overline{e_{z}}+\bar{\Upsilon}_{q} \\
\underline{\dot{e}}_{z}(t)= & \dot{z}-\underline{\dot{z}}=P\left(A_{q}-L_{q} C_{q}\right) P^{-1} \underline{e}_{z} \\
& +\left[P w-\left(P^{+} \underline{w}-P^{-} \bar{w}\right)\right]+\left|P L_{q}\right| \bar{V} E_{p} \\
& -P L_{q} v=R_{q} \underline{e}_{z}+\underline{\Upsilon}_{q}
\end{aligned}
$$

where

$$
\begin{aligned}
& \bar{\Upsilon}_{q}=\left[\left(P^{+} \bar{w}-P^{-} \underline{w}\right)-P w\right]+\left|P L_{q}\right| \bar{V} E_{p}+P L_{q} v \\
& \Upsilon_{q}=\left[P w-\left(P^{+} \underline{w}-P^{-} \bar{w}\right)\right]+\left|P L_{q}\right| \bar{V} E_{p}-P L_{q} v
\end{aligned}
$$
Similarly to the proof of Theorem 2 , the asymptotic stability of the observer (36) is ensured by using a common Lyapunov function for the errors dynamics $\bar{e}_{z}$ and $\underline{e}_{z}$. Following the same steps, the stability analysis is reduced to the feasibility of the LMI as below:

$$
\begin{aligned}
\mathcal{B}_{2}= & {\left[P\left(A_{q}-L_{q} C_{q}\right) P^{-1^{T}}\right] M } \\
& +M\left[P\left(A_{q}-L_{q} C_{q}\right) P^{-1}\right]^{T}+\frac{1}{\delta_{q}} M \\
= & P^{-1^{T}} A_{q}^{T} P^{T} M+M P A_{q} P^{-1}+\frac{1}{\delta_{q}} M \\
& -P^{-1^{T}} C_{q}^{T} L_{q}^{T} P^{T} M-M P L_{q} C_{q} P^{-1} \prec 0
\end{aligned}
$$

In the case of a single mode (non switched systems), it has been shown in several works that the existence of the solution of the Sylvester equation $P$ is not restrictive. In [21], it has been shown that the Jordan canonical form can also be used and $P$ can be time-varying. In addition, a constant change of coordinates has been proposed in [27]. However, the existence of a common transformation matrix $P$ such that $R_{q}, \forall q \in \mathcal{I}$ are Metzler seems to be difficult. The inequality presented in (40) is a nonlinear inequality. Therefore, the computation of the observer gains for (36) can not be easily ensured. As a solution, a second method is proposed. The main idea consists in redesigning two conventional observers in the original basis " $x$ ". Then, stability conditions will be given in terms of LMIs.

Consider the following SLS (9) and two point observers

$$
\left\{\begin{array}{c}
\dot{\hat{x}}^{+}=\left(A_{q}-L_{q} C_{q}\right) \hat{x}^{+}+B_{q} u+P_{q}^{-1}\left(P_{q}^{+} \bar{w}+P_{q}^{-} \bar{w}\right) \\
+L_{q} y_{m}+P_{q}^{-1}\left|P_{q} L_{q}\right| \bar{V} E_{p} \\
\dot{\hat{x}}^{-}=\left(A_{q}-L_{q} C_{q}\right) \hat{x}^{-}+B_{q} u+P_{q}^{-1}\left(-P_{q}^{+} \bar{w}-P_{q}^{-} \bar{w}\right) \\
+L_{q} y_{m}-P_{q}^{-1}\left|P_{q} L_{q}\right| \bar{V} E_{p}
\end{array}\right.
$$

with $P_{q}, q \in \mathcal{I}$, are chosen as in the following theorem and

$$
\left\{\begin{array}{l}
\hat{x}_{0}^{+}=Q_{q}\left(P_{q}^{+} \bar{x}_{0}-P_{q}^{-} \underline{x}_{0}\right) \\
\hat{x}_{0}^{-}=Q_{q}\left(P_{q}^{+} \underline{x}_{0}-P_{q}^{-} \bar{x}_{0}\right)
\end{array}\right.
$$

with $Q_{q}=P_{q}^{-1}$.

The observer (41) is not an interval observer for (9) and its structure is similar to the one in [4] proposed for the case of non switched systems. However, the estimates computed by (41) are used in Theorem 5 to deduce an interval estimation.

Theorem 5 Consider matrices $P_{q}(q \in \mathcal{I})$ such that $F_{q}=P_{q}\left(A_{q}-L_{q} C_{q}\right) P_{q}^{-1}$ are Metzler. If the initial condition $x_{0}$ verifies $\underline{x}_{0} \leq x_{0} \leq \bar{x}_{0}$, then an interval estimation for (9) is given by:

$$
\left\{\begin{array}{l}
\underline{x}=Q_{q}{ }^{+} P_{q} \hat{x}^{-}-Q_{q}{ }^{-} P_{q} \hat{x}^{+} \\
\bar{x}=Q_{q}{ }^{+} P_{q} \hat{x}^{+}-Q_{q}{ }^{-} P_{q} \hat{x}^{-}
\end{array}\right.
$$

satisfying

$$
\underline{x}(t) \leq x(t) \leq \bar{x}(t)
$$

In addition, if there exists $M=M^{T} \succ 0$ such that

$$
A_{q}^{T} M+M A_{q}-C_{q}^{T} W_{q}^{T}-W_{q} C_{q}+\sigma_{q} M \prec 0
$$

where

$$
\sigma_{q}=\frac{3}{\delta_{q}} \text { and } W_{q}=M L_{q}
$$

then (41) is asymptotically stable and $\bar{x}, \underline{x}$ are bounded.

PROOF. Consider the errors $E_{q}^{+}=P_{q} \hat{x}^{+}-P_{q} x$ and $E_{q}^{-}=P_{q} x-P_{q} \hat{x}^{-}$. Let us show that $\bar{x}-x \geq 0$ and $x-\underline{x} \geq 0$ where $\bar{x}$ and $\underline{x}$ are computed by (43) and (41). From (9) and (41) the dynamics of the errors $E_{q}^{+}$and $E_{q}^{-}$are given by:

$$
\begin{aligned}
\dot{E}_{q}^{+}= & P_{q} \dot{\hat{x}}^{+}-P_{q} \dot{x} \\
= & P_{q}\left(A_{q}-L_{q} C_{q}\right) \hat{x}^{+}-P_{q}\left(A_{q}-L_{q} C_{q}\right) x \\
& +\left[\left(P_{q}^{+} \bar{w}+P_{q}^{-} \bar{w}\right)-P_{q} w\right]+P_{q} L_{q} v+\left|P_{q} L_{q}\right| \bar{V} E_{p} \\
= & P_{q}\left(A_{q}-L_{q} C_{q}\right) P_{q}^{-1}\left(P_{q} \hat{x}^{+}-P_{q} x\right)+\gamma_{q}^{+} \\
= & F_{q} E_{q}^{+}+\gamma_{q}^{+} \\
\dot{E}_{q}^{-}= & P_{q} \dot{x}-P_{q} \dot{\hat{x}}^{-}=F_{q} E_{q}^{-}+\gamma_{q}^{-}
\end{aligned}
$$

where

$\gamma_{q}^{+}=\left[\left(P_{q}^{+} \bar{w}+P_{q}^{-} \bar{w}\right)-P_{q} w\right]+P_{q} L_{q} v+\left|P_{q} L_{q}\right| \bar{V} E_{p}$

$\gamma_{q}^{-}=\left[P_{q} w-\left(-P_{q}^{+} \bar{w}-P_{q}^{-} \bar{w}\right)\right]+\left|P_{q} L_{q}\right| \bar{V} E_{p}-P_{q} L_{q} v$

According to Lemma 3 we have $-P_{q}^{+} \bar{w}-P_{q}^{-} \bar{w} \leq P_{q} w \leq$ $P_{q}^{+} \bar{w}+P_{q}^{-} \bar{w}$. Since $P_{q}\left(A_{q}-L_{q} C_{q}\right) P_{q}^{-1}$ are assumed to be Metzler, and by construction $\gamma_{q}^{+}$and $\gamma_{q}^{-}$are nonnegative for all $t \geq 0$. Then, if $\bar{x}_{0}$ and $\underline{x}_{0}$ are chosen such that $E_{q}^{+}(0)$ and $E_{q}^{-}(0)$ are nonnegative, then the errors $E_{q}^{+}(t)$ and $E_{q}^{-}(t)$ stay nonnegative $\forall t \geq 0$ such that $P_{q} \hat{x}^{-} \leq P_{q} x \leq P_{q} \hat{x}^{+}$. As $Q_{q}=P_{q}^{-1}$, then by using Lemma 3, it follows that $\underline{x}=Q_{q}{ }^{+} P_{q} \hat{x}^{-}-Q_{q}{ }^{-} P_{q} \hat{x}^{+}$and 
$\bar{x}=Q_{q}{ }^{+} P_{q} \hat{x}^{+}-Q_{q}{ }^{-} P_{q} \hat{x}^{-}$which means that $\underline{x} \leq x \leq$ $\bar{x}, \forall t \geq 0$.

For the stability analysis, let us now show that $E_{q}^{+}$ and $E_{q}^{-}$are asymptotically stable or simply show that $\left(\hat{x}^{+}-x\right)$ and $\left(x-\hat{x}^{-}\right)$are asymptotically stable. Let $e^{+}=\left(\hat{x}^{+}-x\right)$ and $e^{-}=\left(x-\hat{x}^{-}\right)$be the observation errors and consider the following Lyapunov function:

$$
V\left(e^{+}\right)=e^{+^{T}} M e^{+}
$$

where $M=M^{T} \succ 0$. As in the proof of Theorem 2, the derivative of the Lyapunov function (48) is given by:

$$
\begin{aligned}
\dot{V}\left(e^{+}\right)= & \dot{e}^{{ }^{T}} M e^{+}+e^{+^{T}} M \dot{e}^{+} \\
= & e^{+T}\left[\left(A_{q}-L_{q} C_{q}\right)^{T} M+M\left(A_{q}-L_{q} C_{q}\right)\right] e^{+} \\
& +2 e^{+^{T}} M P_{q}{ }^{-1}\left(P_{q}^{+} \bar{w}+P_{q}^{-} \bar{w}\right)-2 e^{+^{T}} M w \\
& +2 e^{+^{T}} M L_{q} v+2 e^{+^{T}} M\left|L_{q}\right| \bar{V} E_{p}
\end{aligned}
$$

According to Lemma 1, we have

$$
\dot{V}\left(e^{+}\right) \leq e^{+^{T}} \mathcal{B}_{3} e^{+}+\mathcal{C}_{5}
$$

where $\mathcal{B}_{3}=\left(A_{q}-L_{q} C_{q}\right)^{T} M+M\left(A_{q}-L_{q} C_{q}\right)+\frac{3}{\delta_{q}} M$

$$
=A_{q}{ }^{T} M+M A_{q}-C_{q}{ }^{T} W_{q}{ }^{T}-W_{q} C_{q}+\frac{3}{\delta_{q}} M
$$

and $\mathcal{C}_{5}=\bar{w}^{T}\left[\delta_{q} P_{q}^{+^{T}} P_{q}^{-1^{T}} M P_{q}^{-1} P_{q}^{+}\right] \bar{w}$

$$
\begin{aligned}
& +\bar{w}^{T}\left[\delta_{q} P_{q}^{-T} P_{q}^{-1^{T}} M P_{q}^{-1} P_{q}^{-}\right] \bar{w}-w^{T}\left[\delta_{q} M\right] w \\
& +E_{p}^{T}\left[\delta_{q} \bar{V}\left|L_{q}\right|^{T} M\left|L_{q}\right| \bar{V}\right] E_{p}+v^{T}\left[\delta_{q} L_{q}^{T} M L_{q}\right] v
\end{aligned}
$$

The noise $v$ and disturbance $w$ are bounded, it follows that $\mathcal{C}_{5}$ is bounded. Therefore, if $\mathcal{B}_{3} \prec 0$, the observation error $e^{+}$is bounded. The same arguments allow one to show that the observation error $e^{-}$is also bounded. In addition, since $P_{q}$ and $Q_{q}$ are bounded for all $(q \in \mathcal{I})$ then $E_{q}^{+}$and $E_{q}^{-}$are bounded.

The cooperativity property has motivated the need for state transformation. However, computing a transformation matrix $P_{q}$ for each subsystem, such that $z_{q}=P_{q} x$, transforms the switched system (9) into a hybrid one. Besides, the stability analysis in the basis " $z$ " seems to be difficult. The existence of a non singular common matrix transforming (10) into a cooperative form is not always ensured. These limitations prove the interest of the methodology proposed above.

\section{Numerical example}

\subsection{Example 1: Trotter approximation}

Consider the system (22) with two subsystems and

$$
A_{1}=\left[\begin{array}{ccc}
-4.0 & 2.0 & 1.0 \\
-1.0 & -3.0 & 2.0 \\
0 & -0.5 & -2.0
\end{array}\right], A_{2}=\left[\begin{array}{ccc}
-1.0 & -1.0 & -0.5 \\
1.0 & -4.0 & -1.0 \\
3.0 & 1.0 & -3.0
\end{array}\right],
$$

$$
\begin{gathered}
B_{1}=B_{2}=\left[\begin{array}{lll}
1.2 & -1.5 & 1
\end{array}\right]^{T}, C_{1}=C_{2}=\left[\begin{array}{lll}
1 & 0 & 0
\end{array}\right], \\
v(t)=\sin (10 t) \bar{V}, \quad \text { with } \bar{V}=1
\end{gathered}
$$

and $w(t)=[\sin (2 t) 1.5 \sin (3 t) \cos (2 t)]^{T}$, which satisfy $-\bar{w}(t) \leq w(t) \leq \bar{w}(t)$ where $\bar{w}=\left[\begin{array}{lll}1 & 1.5 & 1\end{array}\right]^{T}$.

For the cooperativity of estimated error bounds, the parameter $m=2$ in the expression (28) guarantees the nonnegativity of $\Delta$ and the Assumption 3 is fulfilled due to the matrix $A_{1}-L_{1} C_{1}+A_{2}-L_{2} C_{2}$ is Metzler where $L_{1}=\left[\begin{array}{lll}0.0 & -0.5 & 1.0\end{array}\right]^{T}, L_{2}=\left[\begin{array}{lll}3 & -1 & 1\end{array}\right]^{T}$. The time of activation of each subsystem is $\tau=0.125$.

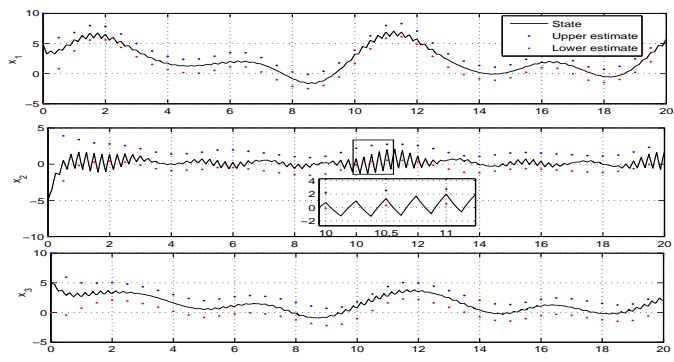

Fig. 1. Simulation results of the first example.

In addition to that, the stability of discrete interval observer (29) is verified by satisfying the matrix inequality (30) in the Assumption 4.

The result of simulation is presented in Fig. 1 where dots correspond to the estimated lower and upper bounds and the solid lines correspond to the actual state. The input signal used in simulation is $u(t)=4+10 \sin (t) \cos (0.3 t)$. This simulation result shows that the developed observer can estimates an interval of admissible values of the state in discrete time instants.

\subsection{Example 2: Transformation of coordinates}

Consider now the system (9) with $\mathcal{I}=\overline{1,2}$ and

$$
\begin{aligned}
& A_{1}=\left[\begin{array}{ccc}
-3 & 0 & 0.5 \\
0 & -2.754 & 0 \\
0 & 0 & -1
\end{array}\right], A_{2}=\left[\begin{array}{ccc}
-2.5 & 0 & 0.2 \\
0 & -0.452 & 0 \\
-1 & 0 & -1
\end{array}\right] \\
& B_{1}=B_{2}=\left[\begin{array}{lll}
1 & 1 & 0
\end{array}\right]^{T}, C_{1}=\left[\begin{array}{lll}
0 & 0 & 1
\end{array}\right], C_{2}=\left[\begin{array}{lll}
0 & 1 & 0
\end{array}\right]
\end{aligned}
$$

$w(t)$ and $v(t)$ are uniformly distributed bounded signals such that $-\bar{w} \leq w(t) \leq \bar{w}$ with $\bar{w}=$ $\left[\begin{array}{lll}0.015 & 0.015 & 0.015\end{array}\right]^{T}$ and $-\bar{V} E_{p} \leq v(t) \leq \bar{V} E_{p}$ with $\bar{V}=0.05$.

Using the Matlab LMI toolbox, it was not possible to find a solution for (12). Therefore, the interval observer (11) cannot be applied. In addition, it is not trivial to compute a common matrix $P$ such that $P\left(A_{q}-L_{q} C_{q}\right) P^{-1}$ 
are Metzler. However, the interval observer (41)-(43) can be used with different $P_{1}, P_{2}$ transformation matrices. Now, using the Matlab LMI toolbox, one can solve the LMI defined by (45). One feasible solution is given by: $L_{1}=\left[\begin{array}{lll}0.9226 & 0 & -0.0111\end{array}\right]^{T}, L_{2}=\left[\begin{array}{lll}0 & 1.9007 & 0\end{array}\right]^{T}$. Note that $P_{q}$ are computed such that $P_{q}\left(A_{q}-L_{q} C_{q}\right) P_{q}^{-1}$ are Metzler for all $q \in \overline{1,2}$. All conditions of Theorem 5 are satisfied; it follows that the system (41) is asymptotically stable and the bounds $\bar{x}, \underline{x}$ are computed using (43) with $Q_{q}=P_{q}^{-1}$.

The results of simulation of the obtained observer are depicted in Fig. 2 where solid lines present the state and dashed lines present the estimated bounds. The input signal $u(t)$ is a square signal with frequency $2 \mathrm{~Hz}$ and magnitude 0.5 . The switching between the two subsystems is governed by the switching signal plotted in Fig. 3. The interval observer (41)-(43) is initialized by $\hat{x}_{0}^{+}$and $\hat{x}_{0}^{-}$which are computed by (42) where $-\bar{x}_{0} \leq x_{0} \leq \bar{x}_{0}$ with $\bar{x}_{0}=\left[\begin{array}{lll}0.2 & 0.2 & 0.2\end{array}\right]^{T}$.

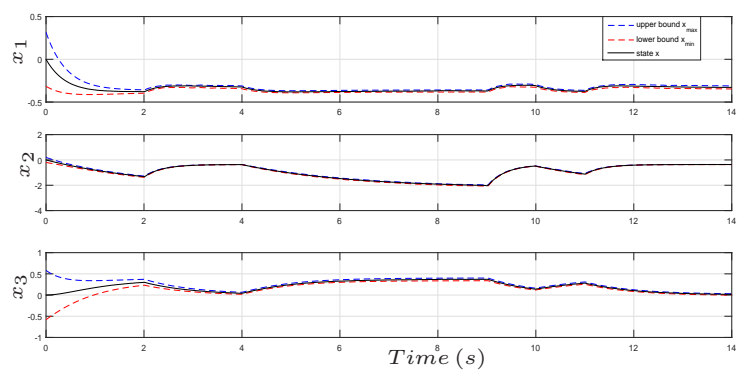

Fig. 2. Simulation results of the second example.

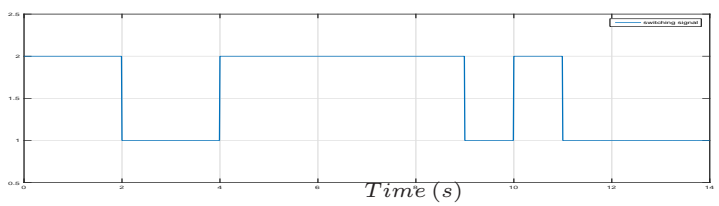

Fig. 3. Switching signal.

The results show that, despite the disturbances, the state is always inside the upper and the lower trajectories. The interval observer has exhibited approved stability properties. The relation $\underline{x}(t) \leq x(t) \leq \bar{x}(t), \forall t \geq 0$ is always verified. As shown in Fig. 2, the interval is quite large at the beginning, although its width decreases despite the uncertainties on the measurements. Finally, the interval observer remains stable despite the switching instants.

\section{Conclusion}

This paper focused on state estimation for switched linear systems subject to disturbances in a bounded error context. The disturbances are assumed to belong to certain known intervals at any moment of time. Several methods were proposed to compute a set of guaranteed lower and upper bounds that contain all the admissible values of the state vector. In the first contribution, the cooperativity property is ensured via an accurate computation of observer gains. In the second contribution, an interval observer is designed under change of coordinates. The observer gains are computed by solving LMIs. Efficiency of the proposed methods is demonstrated on numerical examples. In this work, the results have been obtained under restrictive hypothesis, like availability of the instants of switching or existence of a common Lyapunov function, which can be relaxed in forthcoming works. Other promising directions of future works include control design based on interval estimates, and a combination of continuous and discrete-time estimates obtained in this paper.

\section{References}

[1] Fayssal Arichi, Mohamed Djemai, Brahim Cherki, and Noureddine Manamanni. Continuous and discrete state estimation for a class of nonlinear switched systems. IEEE Trans. On Circuits and Systems II, 62(7):691-695, 2015.

[2] Stanislav Chebotarev, Denis Efimov, Tarek Raïssi, and Ali Zolghadri. Interval observers for continuous-time lpv systems with 11/12 performance. Automatica, 58:82-89, 2015.

[3] K.H. Degue, Denis Efimov, and Jean-Pierre Richard. Interval observers for hybrid linear systems. In 10th IFAC Symposium on Nonlinear Control Systems (NOLCOS), Monterey, 2016.

[4] T. N. Dinh, F. Mazenc, and S. I. Niculescu. Interval observer composed of observers for nonlinear systems. In European Control Conference, pages 660-665, 2014.

[5] Mohamed Djemai and Michael Defoort. Hybrid Dynamical Systems. Springer, 2015.

[6] Denis Efimov, Wilfrid Perruquetti, Tarek Raïssi, and Ali Zolghadri. Interval observers for time-varying discrete-time systems. IEEE Transactions on Automatic Control, 2013.

[7] Denis Efimov and Tarek Rassi. Design of interval observers for uncertain dynamical systems. Automation and Remote Control, 77(2):191-225, 2016.

[8] Haifa Ethabet, Tarek Rassi, Messaoud Amairi, and Mohamed Aoun. Interval observers design for continuous-time switched systems. In Proc. 20th IFAC WC 2017, 2017.

[9] Lorenzo Farina and Sergio Rinaldi. Positive Linear Systems: Theory and Applications, volume 50. John Wiley \& Sons, 2000.

[10] Jean-Luc Gouzé, A Rapaport, and Mohamed Zakaria HadjSadok. Interval observers for uncertain biological systems. Ecological modelling, 133(1):45-56, 2000.

[11] G.H. Hardy, J.E. Littlewood, and G. Pólya. Inequalities. Cambridge Mathematical Library. Cambridge University Press, 1952.

[12] Zhongwei He and Wei Xie. Interval state observer for nonlinear switched systems with average dwell time. In 34th IEEE Chinese Control Conference (CCC), pages 2285-2288, 2015.

[13] Zhongwei He and Wei Xie. Control of non-linear switched systems with average dwell time: interval observer-based framework. IET Control Theory \& Applications, 10(1):1016,2016 . 
[14] Laurentiu Hetel. Robust stability and control of switched linear systems. PhD thesis, Nancy University, 2007.

[15] Bo Hu, Guisheng Zhai, and Anthony N Michel. Common quadratic lyapunov-like functions with associated switching regions for two unstable second-order lti systems. International Journal of Control, 75(14):1127-1135, 2002.

[16] Sara Ifqir, Naima Ait Oufroukh, Dalil Ichalal, and Said Mammar. Switched interval observer for uncertain continuous-time systems. in Proc. 20th IFAC WC $201 \%$.

[17] Rihab Lamouchi, Messaoud Amairi, Tarek Raïssi, and Mohamed Aoun. Interval observer design for linear parameter-varying systems subject to component faults. In 24th IEEE Mediterranean Conference on Control and Automation (MED), pages 707-712, 2016.

[18] Daniel Liberzon. Switching in systems and control. Springer Science \& Business Media, 2012.

[19] Hai Lin and Panos J Antsaklis. Stability and stabilizability of switched linear systems: a survey of recent results. IEEE Transactions on Automatic control, 54(2):308-322, 2009.

[20] David Luenberger. Observers for multivariable systems. IEEE Transactions on Automatic Control, 11(2):190-197, 1966.

[21] Frédéric Mazenc and Olivier Bernard. Interval observers for linear time-invariant systems with disturbances. Automatica, 47(1):140-147, 2011.

[22] Frederic Mazenc, Thach Ngoc Dinh, and Silviu Iulian Niculescu. Interval observers for discrete-time systems. International Journal of Robust and Nonlinear Control, 24(17):2867-2890, 2014.

[23] Nacim Meslem and Nacim Ramdani. Interval observer design based on nonlinear hybridization and practical stability analysis. International Journal of Adaptive Control and Signal Processing, 25(3):228-248, 2011.

[24] Henryk Minc. Nonnegative matrices. Wiley, 1988.

[25] W Mitkowski. Dynamical properties of metzler systems. Technical Sciences, 56(4), 2008.

[26] Djahid Rabehi, Denis Efimov, and Jean-Pierre Richard. Interval estimation for linear switched system. In Proc. 20th IFAC WC 2017, 2017.

[27] Tarek Raïssi, Denis Efimov, and Ali Zolghadri. Interval state estimation for a class of nonlinear systems. IEEE Transactions on Automatic Control, 57(1):260-265, 2012.

[28] Tarek Raïssi, Gaétan Videau, and Ali Zolghadri. Interval observer design for consistency checks of nonlinear continuous-time systems. Automatica, 46(3):518-527, 2010.

[29] Héctor Ríos, Diego Mincarelli, Denis Efimov, Wilfrid Perruquetti, and Jorge Davila. Continuous and discrete state estimation for switched lpv systems using parameter identification. Automatica, 62:139-147, 2015.

[30] Masuo Suzuki. Generalized trotter's formula and systematic approximants of exponential operators and inner derivations with applications to many-body problems. Communications in Mathematical Physics, 51(2):183-190, 1976.

[31] Aneel Tanwani, Hyungbo Shim, and Daniel Liberzon. Observability for switched linear systems: characterization and observer design. IEEE Transactions on Automatic Control, 58(4):891-904, 2013.

[32] Hale F Trotter. On the product of semi-groups of operators. Proceedings of the American Mathematical Society, 10(4):545-551, 1959.

[33] Linh Vu, Debasish Chatterjee, and Daniel Liberzon. Inputto-state stability of switched systems and switching adaptive control. Automatica, 43(4):639-646, 2007.
[34] Xudong Zhao, Hao Liu, Junfeng Zhang, and Hongyi Li. Multiple-mode observer design for a class of switched linear systems. IEEE Transactions on Automation Science and Engineering, 12(1):272-280, 2015.

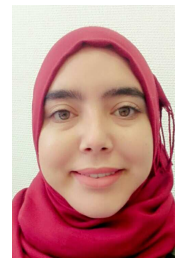

Haifa Ethabet was born in 1991, in Tunisia. She received the Electrical and Automatic Engineering degree from the National Engineering School of Gabes, Tunisia in 2015. She is currently a Ph.D. student in Electrical Engineering and she is a member of the Modeling, Analysis and Control of Systems (MACS) laboratory. Her current research interests include switched systems estimation, interval analysis, and fault detection and isolation.

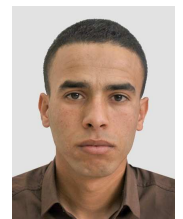

Djahid Rabehi was born in 1990, in Algeria. He earned the Master's Degree from the University of Science and Technology of Lille, France, in 2016. He is currently pursuing the Ph.D. degree in Automatic Control at the University of Orléans, France. His research interests include set-membership estimation, event-triggered estimation, hybrid systems.

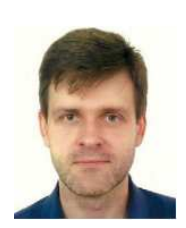

Denis Efimov received the Ph.D. degree in Automatic Control from the Saint-Petersburg State Electrical Engineering University (Russia) in 2001, and the Dr.Sc. degree in Automatic control in 2006 from Institute for Problems of Mechanical Engineering RAS (SaintPetersburg, Russia). From 2000 to 2009 he was research fellow of the Institute for Problems of Mechanical Engineering RAS, Control of Complex Systems Laboratory. From 2006 to 2011 he was working in the LSS (Supelec, France), the Montefiore Institute (University of Liege, Belgium) and the Automatic control group at IMS lab (University of Bordeaux I, France). Since 2011 he joined the Non-A team at Inria Lille center. He is a member of several IFAC TCs and a Senior member of IEEE. His main research interests include nonlinear oscillation analysis, observation and control, switched and nonlinear system stability..

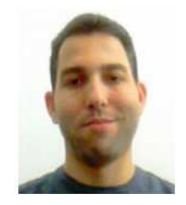

Tarek Rassi received the Engineering degree from École Nationale d'Ingnieurs de Tunis in 2000, the DEA in Automatic Control from École Centrale de Lille in 2001, the Ph.D. degree from the University of Paris XII in 2004 and the Accreditation to Supervise/Conduct Research (HDR) from the University of Bordeaux 1 in 2012. From 2005 to 2011 he was an Associate Professor at the University of Bordeaux 1. Currently, he is a Full Professor at the Conservatoire National des Arts et Métiers, Paris, France. He is a member of the IFAC Technical Committee Modelling, Identification and Signal Processing and a Senior member of IEEE. His research interests include fault detection and isolation, nonlinear systems estimation and robust control. 